Technical Report No. 04/05, August 2005 SMOOTH ESTIMATION OF SURVIVAL FUNCTIONS UNDER MEAN RESIDUAL LIFE ORDERING

Yogendra P. Chaubey and Haipeng Xu 


\title{
Smooth Estimation of Survival Functions under Mean Residual Life Ordering
}

\author{
Yogendra P. Chaubey and Haipeng Xu \\ Concordia University, Montréal, Quebec, Canada
}

\begin{abstract}
Let $X$ and $Y$ be random variables denoting life times with $S_{1}, S_{2}$ denoting their survival functions and $M_{1}, M_{2}$ their mean residual life (MRL) functions, respectively - $X$ is said to be smaller than $Y$ in mean residual life order, if and only if $M_{1}(x) \leq$ $M_{2}(x)$ for all $x$; or equivalently, if $\int_{t}^{\infty} S_{1}(x) d x / \int_{t}^{\infty} S_{2}(x) d x$ is non-increasing over $\left\{t: \int_{t}^{\infty} S_{2}(x) d x>0\right\}$. In this paper we adapt the technique of Chaubey and Sen (1996) to propose smooth estimators of MRL and survival functions based on the estimator considered by $\mathrm{Hu}$ et al. (2002). In the process, we have proposed a new estimator based on the alternative definition and shown that smoothing process carries over large sample properties such as strong consistency. Furthermore, a simulation study demonstrates that new estimators may have better mean square error properties in tails.
\end{abstract}

\section{Introduction}

The mean residual life (MRL) function $M=\{M(x), x \geq 0\}$ corresponding to a non-negative random variable $X$, which denotes life time of a subject or a component is defined as

$$
M(x)=E(X-x \mid X>x)=E(X \mid X>x)-x
$$

It denotes the expected remaining life for the random variable $X$ after survival up to time $x$, and plays an important role in describing the ageing process and therefore has important applications in many fields such as manufacturing, biomedical sciences and actuarial science just to name a few. Guess and Proschan (1988) have given an extensive review for the MRL 
function in reliability theory, where as, more recently, Embrechts et al. (1997) have provided a detailed discussion and statistical applications.

Denoting the survival function of $X$ by $S(x)$, the MRL function (MRLF) can be written as

$$
M(x)=\frac{\int_{x}^{\infty} S(u) d u}{S(x)} I(S(x)>0) .
$$

A distribution is characterized by its MRLF due to the following relation

$$
S(x)=\frac{M(0)}{M(x)} \mathrm{e}^{-\int_{0}^{x}(1 / M(u)) d u}
$$

Yang (1978) proposed estimating $M(x)$, replacing the survival function in Eq. (1.2) by the empirical survival function. The reader may refer to Csörgo and Zitikis (1996) and references therein for the vast literature on the properties of this estimator. Due to the discontinuities inherent in the above estimator, several authors have considered smooth estimators. Ruiz and Guillamòn (1996) use kernel smoothing to estimate the integral in Eq. (1.2), while estimating the denominator by the empirical survival function, where as, Chaubey and Sen (1999) study the properties of the estimator obtained by substituting a smooth estimator of the survival function in its place in Eq. (1.2) based their paper[Chaubey and Sen (1996)]. Recently, Abdous and Berred (2005) have studied the properties of local polynomial based estimator of $m(x)$ obtained through kernel smoothing of Yang's estimator.

$\mathrm{Hu}$ et al. (2002) argue that "in some cases, particularly in health sciences and actuarial sciences, the MRLF gives a more intuitive picture of survival or aging than the survival function or the hazard rate function," $h(x)=f(x) / S(x)$, where $f(x)$ denotes the density corresponding to $S(x)$. Let $X$ and $Y$ be two random variables with finite means, representing life times of two populations with survival functions $S_{1}$ and $S_{2}$ and MRLFs $m_{1}$ and $m_{2}$. When confronted with the problem of comparing two populations to see which one has longer life, the researcher has various choices. One may compare just the two means, i.e. $m_{1}(0)$ and $m_{2}(0)$, or rather than basing the decision on a single point, one could compare $X$ and $Y$ under stochastic ordering restriction i.e. $S_{1}(x) \leq(\geq) S_{2}(x)$ for all $x$. Since, these comparisons do not take into account the age of the components, a more meaningful method may be to compare the conditional distributions given survival up to age $x$ for both the components. The above ordering being a very strong one, Hu et al. (2002) recommend comparing $X$ and $Y$ with respect to their MRLFs. Ebrahimi (1993) was the first one to consider estimating the MRLFs under MRL order, although on a compact set $\left(t_{1}, t_{2}\right)$. Hu et al. (2002) modified Ebrahimi's estimators to assure that the resulting estimators are indeed MRLF's and studied the asymptotic and finite sample properties of their estimators. 
It is to be noted that the estimators of the corresponding survival functions can be obtained via the relation in Eq. (1.3), the discontinuities in the resulting estimators remain. In life sciences and industrial situations, where smooth estimators of survival functions are desired, this is not an attractive proposition when the underlying distributions are assumed to be continuous. In such situations many applied practitioners [see Kim and Proschan (1991)] would prefer to have smooth estimators and as such there is a lot of interest in smooth estimation of survival functions (see Chaubey and Kochar (2000) and references therein). One simple way to obtain smooth estimators is to smooth the estimators obtained from Eq. (1.3), but the resulting smooth estimators may not preserve the mean residual life order. As such our purpose in this paper is to propose smooth estimators of the survival function under the mean residual life restriction. The set up considered here is that of uncensored data, however, the method can be easily generalized to the case of randomly censored data in light of the discussion in Chaubey and Sen (1998).

The organization of the paper is as follows. Section 2 presents the formal definition of MRL ordering and its relation to other stochastic orders. Section 3 presents the estimators and section 4 gives some asymptotic properties. The final section presents the results of a simulation study comparing the proposed estimators.

\section{Preliminaries}

In the following discussion we will consider random variables $(X, Y)$, with their density, survival, hazard and MRL functions given by $\left(f_{1}, f_{2}\right),\left(S_{1}, S_{2}\right),\left(h_{1}, h_{2}\right)$ and $\left(m_{1}, m_{2}\right)$, respectively.

Definition 2.1 Let $X$ and $Y$ be two random variables with finite means whose corresponding survival functions and $M R L$ functions are $S_{1}$ and $S_{2}, M_{1}$ and $M_{2}$, respectively. The random variable $X$ is said to be smaller than $Y$ in the mean residual life order (denoted as $X \leq{ }_{m r l} Y$ ), if

$$
M_{1}(x) \leq M_{2}(x), \text { for all } x>0
$$

Since, the MRL function $M$ for a random variable $X$ can be written in terms of its hazard rate function $h$ as

$$
M(x)=\int_{x}^{\infty} \exp \left\{-\int_{x}^{u} h(t) d t\right\} d u
$$


Thus

$$
h_{1}(x) \geq h_{2}(x) \Rightarrow M_{1}(x) \leq M_{2}(x), \forall x>0 .
$$

This shows that MRL ordering is weaker than hazard rate ordering (also known as the uniform stochastic ordering). We defer the discussion on different partial orderings and their applications, to the book by Shaked and Shanthilumar (1994).

Noting that

$$
M_{1}(x) \leq M_{2}(x) \Leftrightarrow \frac{d}{d x} \frac{W_{1}(x)}{W_{2}(x)} \leq 0,
$$

where $W_{i}(x)=\int_{x}^{\infty} S_{i}(u) d u, i=1,2$. we can obtain the following alternative definition of MRL order;

Definition 2.2 Under the conditions of Def. 2.1, $X \leq_{m r l} Y$ if, and only if,

$$
\frac{\int_{t}^{\infty} S_{1}(x) d x}{\int_{t}^{\infty} S_{2}(x) d x} \text { is non-increasing in } t \text { over }\left\{t: \int_{t}^{\infty} S_{2}(x) d x>0\right\} \text {. }
$$

Now we discuss the estimators of the survival functions under MRL order. We consider the order $M_{1}(x) \leq M_{2}(x), \forall x$. The complementary case can be treated by symmetry. Let $\hat{S}_{1}$ and $\hat{S}_{2}$ denote the empirical survival functions for $x$ and $Y$ respectively based on independent samples of sizes $n_{1}$ and $n_{2}$ respectively. The empirical estimators of $M_{i}, i=1,2$ are obtained using the formula (Yang, 1978),

$$
M_{i}(x)=\frac{\int_{x}^{\infty} \hat{S}_{i}(u) d u}{\hat{S}_{i}(u)} I\left(\hat{S}_{i}(x)>0\right), i=1,2 .
$$

Note that the above expressions can be simplified in terms of the order statistics of the corresponding sample, as for a random sample $T_{1}, T_{2}, \ldots, T_{n}$, the empirical MRLF is given by

$$
\hat{M}(t)=\left\{\begin{array}{cl}
\frac{1}{n-k} \sum_{i=k+1}^{n}\left(T_{n: i}-t\right) & \text { for } T_{n: k} \leq t<T_{n: k+1}, k<n \\
0 & \text { for } t \geq T_{n: n}
\end{array}\right.
$$

where $T_{i: n}$ denotes the $i^{\text {th }}$ - order statistic from the random sample. First, we give the form of the estimators considered in $\mathrm{Hu}$ et al. (2002). For the single sample case, suppose that $M_{2}$ is known and $M_{1}(x) \leq M_{2}(x)$, the proposed estimator of $M_{1}(x)$ is given by

$$
M_{1}^{*}(x)=\hat{M}_{1}(x) \wedge M_{2}(x) .
$$


On the other hand, for the two sample case, when $M_{1}$ and $M_{2}$, both are considered unknown, the estimators of $M_{i}(x), i=1,2$ are given by

$$
M_{1}^{*}(x)=\hat{M}_{1}(x) \wedge \hat{M}(x), \quad M_{2}^{*}(x)=\hat{M}_{1}(x) \vee \hat{M}(x),
$$

where $\hat{M}(x)$, is given by

$$
\hat{M}(x)=\hat{w}_{1}(x) \hat{M}_{1}(x)+\hat{w}_{2}(x) \hat{M}_{2}(x),
$$

with $\hat{w}_{i}(x)$ being given by

$$
\hat{w}_{i}(x)=\frac{n_{i} \hat{S}_{i}(x)}{n_{1} \hat{S}_{1}(x)+n_{2} \hat{S}_{2}(x)}
$$

For the reverse order, i.e. $M_{1} \geq M_{2}$, the estimators are defined as

$$
M_{1}^{*}(x)=\hat{M}_{1}(x) \vee M_{2}(x)
$$

and

$$
M_{1}^{*}(x)=\hat{M}_{1}(x) \vee \hat{M}(x), \quad M_{2}^{*}(x)=\hat{M}_{1}(x) \wedge \hat{M}(x) .
$$

Before we present the smooth estimators, we present the following results about the estimators $M_{i}^{*}(x), i=1,2$ as established in $\mathrm{Hu}$ et al. (2002).

Proposition 2.1 Let the support of $S_{i}$ be given by $\left[0, b_{i}\right)$, where possibly $b_{i}=\infty$. Let, $\|f\|_{a}^{b}$ denote $\sup _{a \leq x \leq b}|f(x)|$, then we have for $0 \leq b<b_{i}$,

$$
\left\|M_{i}^{*}-M_{i}\right\|_{0}^{b} \rightarrow 0 \text { a.s. }
$$

where the limit is according to as $n_{1} \rightarrow \infty$ or $n_{1}, n_{2} \rightarrow \infty$ as the case may be.

We would like to remark that, in what follows, we consider $b_{i}=\infty, i=1,2$ as the smooth estimators are appropriate for this case. However, in case the support is finite, we can modify the smooth estimator as follows. Suppose, $\tilde{S}(x)$ is a smooth survival function defined on $[0, \infty)$, a modified smooth estimator on $[0, b)$ is given by

$$
\tilde{S}^{*}(x)= \begin{cases}\frac{\tilde{S}(x)-\tilde{S}(b)}{1-\tilde{S}(b)} & \text { for } x<b \\ 0 & \text { for } x \geq b .\end{cases}
$$




\section{Smooth Estimators of MRLFs and Survival Func- tions under MRL Ordering}

The key to proposing the smooth estimators is the following so called Hille's(1948) lemma [see Feller (1965), pp. 219]

Lemma 3.1 : For any continuous and bounded function $u$, defined on $R^{+}$let

$$
u^{*}(x)=\sum_{i=1}^{\infty} p_{k}(\lambda x) u(k / \lambda),
$$

where

$$
p_{k}(t)=\mathrm{e}^{-t} \frac{t^{k}}{k !}, k=0,1, \ldots
$$

then, as $\lambda \rightarrow \infty$,

$$
\left\|u(x)-u^{*}(x)\right\|_{a}^{b} \rightarrow 0, \text { uniformly }
$$

for all $0 \leq a \leq x \leq b<\infty$. Furthermore, if $u$ is monotone this convergence extends over the whole of $R^{+}$.

Chaubey and Sen(1996) used a modified version of the above lemma in proposing smooth estimators of the survival function and the corresponding density, substituting the empirical distribution function in place of $u$. Further, noting that if $u$ is monotone, then so is $u^{*}$, Chaubey and Kochar (2000) proposed a modification of Chaubey-Sen method for estimating survival distributions constrained by stochastic ordering. Here, we use a similar technique for estimating the mean residual life under MRL order and then use Eq. (1.3) to get the smooth estimator of the survival function. The resulting estimators do preserve the MRL order as they have been derived from MRLFs having this order. Another alternative is to use the characterization of MRL order given by Def. 2.5 where we obtain alternative estimators of MRLFs which preserve the MRL ordering and again using these in Eq. (1.3) we get alternative estimators of the survival functions which preserve the MRL ordering.

\subsection{One Sample Case}

\section{Method 1 - Use of Definition 2.4}

Let $V_{n}(x)=M_{2}(x)-M_{1}^{*}(x)$. Let

$$
\tilde{V}_{n}(x)=\sum_{i=0}^{\infty} p_{k}\left(\lambda_{n} x\right) V_{n}\left(k / \lambda_{n}\right) .
$$


Then our smooth estimator of $M_{1}$ is given by

$$
\tilde{M}_{1,1}(x)=M_{2}(x)-\tilde{V}_{n}(x) .
$$

Assuming $M_{2}(x)$ to be bounded, it is clear that $\hat{V}_{n}(x)$ is a bounded nonnegative function, the smooth estimator $M_{1,1}$ preserves the MRL ordering, $M_{1} \leq M_{2}$. It also preserves some other asymptotic properties inherent in $M_{1}^{*}$, as will be seen in Sec. 4. As discussed in Chaubey and Kochar (2000), we select

$$
\lambda_{n}=\frac{n}{X_{n: n}},
$$

which almost surely converges to $\infty$ as $n \rightarrow \infty$, provided $E(X)$ exists.

\section{Method 2 - Use of Definition 2.5}

This method starts with a smooth estimation of

$$
\theta(x)=\frac{W_{1}(x)}{W_{2}(x)}
$$

where $W_{i}(x)=\int_{x}^{\infty} S_{i}(u) d u, i=1,2$. It is simple to obtain a plug-in estimator of $W_{1}(x)$ based on the empirical survival function [see Eq (2.9)],

$$
W_{1 n}(x)=\left\{\begin{array}{cl}
\frac{1}{n} \sum_{i=k+1}^{n}\left(X_{n: i}-x\right) & \text { for } X_{n: k} \leq x<X_{n: k+1}, k<n \\
0 & \text { for } t \geq X_{n: n}
\end{array}\right.
$$

Similar to estimating the ratio of survival functions under USO, as considered by Rojo and Samniego (1993), we estimate $\theta(x)$ by $\hat{\theta}_{n}(x)\left(\bar{\theta}_{n}(x)\right)$ for the case $M_{1} \leq M_{2}\left(M_{1} \geq M_{2}\right)$ as given by

$$
\begin{aligned}
\hat{\theta}_{n}(x) & \left.=\inf _{0 \leq t \leq x} \frac{W_{1 n}(t)}{W_{2}(t)} I\left(W_{2}(x)>0\right)\right) \\
\left(\bar{\theta}_{n}(x)\right. & =\sup _{0 \leq t \leq x} \frac{W_{1 n_{1}}(t)}{W_{2}(t)} I\left(W_{2}(x)>0\right),
\end{aligned}
$$

and corresponding $W_{1}$ estimated by,

$$
\begin{aligned}
\hat{W}_{1 n}(x) & =\hat{\theta}_{n}(x) W_{2}(x) \\
\left(\bar{W}_{1 n}(x)\right. & \left.=\bar{\theta}_{n}(x) W_{2}(x) I\left(W_{2}(x)>0\right)+W_{1 n}(x) I\left(W_{2}(x)=0\right)\right) .
\end{aligned}
$$

The contribution of the second term in the parenthesis in the above set of equations drops out for the case of infinite support and hence will not be further considered. Also, we consider 
the case $M_{1} \leq M_{2}$, in detail. The reverse order can be dealt in a similar fashion. Now, we can use the Chaubey-Sen smooth estimator $\tilde{S}_{1 n}(x)$ of the survival function $S_{1}(x)$, resulting in a smooth estimator of $W_{1 n}(x)$ using the Hille's theorem, thereby giving a smooth estimator of the mean residual life $M_{1}(x)$. But the MRL ordering properties may be lost in this process. However, if we obtain a smooth estimator of $\theta(x)$ by smoothing $\theta_{n}(x)$, the monotonicity is preserved in the smooth estimator and we get a smooth estimator of $W_{1 n}(x)$, as

$$
\tilde{W}_{1 n}(x)=\tilde{\theta}_{n}(x) W_{2}(x),
$$

where

$$
\tilde{\theta}_{n}(x)=\sum_{i=0}^{\infty} p_{k}\left(\lambda_{n} x\right) \hat{\theta}_{n}\left(\frac{k}{\lambda_{n}}\right)
$$

Let us define $\tilde{S}_{1,2}(x)$ by the differential equation

$$
\tilde{S}_{1,2}(x)=-\frac{d}{d x} \tilde{W}_{1 n}(x)
$$

we have

$$
\tilde{S}_{1,2}(x)=-\frac{d}{d x} \tilde{\theta}_{n}(x) W_{2}(x)+S_{2}(x) \tilde{\theta}_{n}(x) .
$$

Furthermore, resulting smooth estimator of $M_{1}$ is given by

$$
\tilde{M}_{1,2}(x)=\frac{\tilde{W}_{1 n}(x)}{\tilde{S}_{1,2}(x)} .
$$

This estimator also preserves the required MRL ordering and provides an alternative smooth estimator of $M_{1}(x)$. A more explicit expression for $\tilde{M}_{1,2}(x)$ is given by

$$
\tilde{M}_{1,2}(x)=\frac{M_{2}(x)}{1-\frac{d}{d x}\left[\log _{\mathrm{e}} \tilde{\theta}_{n}(x)\right] M_{2}(x)},
$$

the derivative of $\tilde{\theta}_{n}(x)$ required in Eqs. (3.8) and (3.9) may be computed as

$$
\frac{d}{d x} \tilde{\theta}_{n}(x)=-\lambda_{n}\left[\sum_{k=0}^{N-1}\left\{\hat{\theta}_{n}\left(\frac{k}{\lambda_{n}}\right)-\hat{\theta}_{n}\left(\frac{k+1}{\lambda_{n}}\right)\right\} p_{k}\left(\lambda_{n} x\right)\right],
$$

where $N$ is the smallest integer such that $\hat{\theta}_{n}\left(k / \lambda_{n}\right)=0$ for $k \geq N$.

Both the methods are easy to use as for as computation of the MRLF's go. However, the first method is more complicated for computing the smooth estimator of survival function since it may require numerical integration. 


\subsection{Two-sample Case}

\section{Method 1 - Use of Definition 2.4}

For the two sample case, we start with the pair of (non-smooth) estimators $\left(M_{1}^{*}, M_{2}^{*}\right)$ as given by Eq. (2.11). We basically obtain a smooth estimator of $M(x)$ and estimate $M_{1}, M_{2}$ as two one-sample problems as above, with the restriction, $M_{1} \leq \hat{M}$, and $M_{2} \geq \hat{M}$ as if $\hat{M}$ is known. Thus, we consider first a smooth estimator

$$
\tilde{M}(x)=\sum_{i=0}^{\infty} p_{k}(\lambda x) \hat{M}(k / \lambda),
$$

of $M(x)$, where, $\lambda \equiv \lambda_{n_{1}, n_{2}}=\min \left(\lambda_{n_{1}}, \lambda_{n_{1}}\right)$, we have dropped the subscript for the ease of notation. The pair of smooth estimators, thus are given by

$$
\tilde{M}_{1,1}(x)=\tilde{M}(x)-\tilde{V}_{1}(x), \tilde{M}_{2,1}(x)=\tilde{M}(x)+\tilde{V}_{2}(x)
$$

where

$$
\tilde{V}_{i}(x)=\sum_{k=0}^{\infty} p_{k}(\lambda x) V_{i}\left(\frac{k}{\lambda}\right),
$$

and

$$
V_{1}(x)=\hat{M}(x)-M_{1}^{*}(x), V_{2}(x)=M_{2}^{*}(x)-\hat{M}(x) .
$$

The smooth estimators of $S_{1}$ and $S_{2}$ are then obtained using Eq. (1.3).

\section{Method 2 - Use of Definition 2.5}

In this approach, we follow the discussion in Mukerjee (1996) for estimation of survival functions under uniform stochastic ordering. Let $b_{\hat{M}}=\sup \{x: \hat{M}(x)>0\}$, we define

$$
\begin{aligned}
& \hat{\theta}_{1}(x)=\inf _{0 \leq t \leq x} \frac{W_{1 n_{1}}(t)}{\hat{W}(t)}, \\
& \hat{\theta}_{2}(x)=\sup _{0 \leq t \leq x} \frac{W_{2 n_{2}}(t)}{\hat{W}(t)},
\end{aligned}
$$

where [see Eq. (2.12)]

$$
\hat{W}(x)=w_{1}(x) W_{1 n_{1}}(x)+w_{2}(x) W_{2 n_{2}}(x) .
$$

Let us denote by $\tilde{\theta}_{1}(x), \tilde{\theta}_{2}(x), \tilde{W}_{1 n_{1}}(x), \tilde{W}_{2 n_{2}}(x)$ smooth versions of the corresponding quantities. Let

$$
\tilde{W}(x)=w_{1}(x) \tilde{W}_{1 n_{1}}(x)+w_{2}(x) \tilde{W}_{2 n_{2}}(x) .
$$


Then, following the heuristic argument of Mukerjee (1996), the pair of smooth estimators of $\left(W_{1}(x), W_{2}(x)\right)$ is given by

$$
\begin{aligned}
& \tilde{W}_{1 n_{1}}^{*}(x)=\tilde{\theta}_{1}(x) \tilde{W}(x), \\
& \tilde{W}_{2 n_{2}}^{*}(x)=\tilde{\theta}_{2}(x) \tilde{W}(x) .
\end{aligned}
$$

Another pair of estimators may be constructed by directly smoothing $\hat{W}(x)=w_{1}(x) \hat{W}_{1}(x)+$ $w_{2}(x) \hat{W}_{2}(x)$. However, we prefer the preceding pair as we require a smooth estimator of $W_{2}(x)$ giving $\tilde{W}_{2 n_{2}}(x)$ which is then used in finding $\tilde{W}_{(x)}$. This provides the following pair of survival functions:

$$
\begin{aligned}
& \tilde{S}_{1,2}(x)=-\frac{d \tilde{\theta}_{1}(x)}{d x} \tilde{W}(x)+\tilde{\theta}_{1}(x) \tilde{S}(x) \\
& \tilde{S}_{2,2}(x)=-\frac{d \tilde{\theta}_{2}(x)}{d x} \tilde{W}(x)+\tilde{\theta}_{2}(x) \tilde{S}_{(x)}
\end{aligned}
$$

The jump discontinuities at $b_{\hat{M}}$ in the above estimators may be removed by considering may be removed because the smooth estimators beyond this point in a continuous way. Assuming that both $S_{1}$ and $S_{2}$ have infinite support, this problem disappears in a natural way. Consequently, the smooth MRLF estimators are:

$$
\begin{aligned}
\tilde{M}_{1,2}(x) & =\frac{\tilde{M}(x)}{1-\frac{d}{d x}\left[\log _{\mathrm{e}} \tilde{\theta}_{1}(x)\right] \tilde{M}(x)} \\
M_{2,2}(x) & =\frac{\tilde{M}(x)}{1-\frac{d}{d x}\left[\log _{\mathrm{e}} \tilde{\theta}_{2}(x)\right] \tilde{M}(x)}
\end{aligned}
$$

The following section studies the consistency properties of the estimators.

\section{Strong Consistency of Smooth Estimators}

We prove consistency for the estimators in one-sample case. The case of two samples is treated similarly.

\subsection{Consistency for $\tilde{M}_{1,1}$ and $\tilde{S}_{1,1}$}

First, we establish strong consistency of the smooth estimator of MRL in one sample case as given in the following theorem. 
Theorem 4.1 If $S_{1}(x)$ is continuous a.e, $\lambda_{n} \rightarrow \infty$, a.s. then

(i)

$$
\sup _{0 \leq x \leq b}\left|\tilde{M}_{1,1}(x)-M_{1}(x)\right| \rightarrow 0 \text { almost surely as } n \rightarrow \infty \text {. }
$$

here, $b$ in $\mathbf{R}^{+}$and $b<B=\min \left(b_{1}, b_{2}\right), b_{i}$ is the support of $S_{i}$.

(ii) Let $b<B$ be such that $M_{1}(b)>0$, then we have

$$
\sup _{0 \leq x \leq b}\left|\tilde{S}_{1,1}(x)-S_{1}(x)\right| \rightarrow 0 \text { almost surely as } n \rightarrow \infty \text {. }
$$

Proof: Since, $V(x)$ is bounded and continuous on $[0, b]$, by Theorem 3.1 we can claim that

$$
\begin{aligned}
\tilde{V}(x) & =\mathrm{e}^{-\lambda_{n} t} \sum_{k=0}^{\infty} V\left(\frac{k}{\lambda_{n}}\right) \frac{\left(\lambda_{n} t\right)^{k}}{k !} \\
& \rightarrow V(x) \text { as } \lambda_{n} \rightarrow \infty
\end{aligned}
$$

uniformly on $[0, b]$. Then we have

$$
\begin{aligned}
\sup _{0 \leq x \leq b}\left|\tilde{V}_{n}(x)-V(x)\right| & =\sup _{0 \leq x \leq b}\left|\tilde{V}_{n}(x)-\tilde{V}(x)+\tilde{V}(x)-V(x)\right| \\
& \leq \sup _{0 \leq x \leq b}\left|\tilde{V}_{n}(x)-\tilde{V}(x)\right|+\sup _{0 \leq x \leq b}|\tilde{V}(x)-V(x)| \\
& \leq \max _{k \leq N}\left|V_{n}\left(\frac{k}{\lambda_{n}}\right)-V\left(\frac{k}{\lambda_{n}}\right)\right|+\sup _{0 \leq x \leq b}|\tilde{V}(x)-V(x)| \\
& \leq \sup _{0 \leq x \leq b}\left|V_{n}(x)-V(x)\right|+\sup _{0 \leq x \leq b}|\tilde{V}(x)-V(x)|
\end{aligned}
$$

The first term converges to zero due to the strong convergence of $M_{1}^{*}$ as established in $\mathrm{Hu}$ et al. (2000) [see Proposition 2.1] and the second term converges to zero from Eq. (4.3). Hence, it follows that

$$
\begin{aligned}
\sup _{0 \leq x \leq b}\left|\tilde{M}_{1,1}(x)-M_{1}(x)\right| & =\sup _{0 \leq x \leq b}\left|M_{2}(x)-\tilde{V}_{n}(x)-M_{1}(x)\right| \\
& =\sup _{0 \leq x \leq b}\left|V(x)-\tilde{V}_{n}(x)\right| \\
& \rightarrow 0 \text { almost surely as } n \rightarrow \infty
\end{aligned}
$$


This establishes the strong consistency of $\tilde{M}_{1,1}$. For proving the consistency of $\tilde{S}_{1,1}$, note that since $\tilde{M}_{1,1}(x)$ and $M_{1}(x)$ are bounded on $[0, b]$ and nonzero, so

$$
\sup _{0 \leq x \leq b}\left|\frac{1}{\tilde{M}_{1,1}(x)}-\frac{1}{M_{1}(x)}\right|=\sup _{0 \leq x \leq b}\left|\frac{M_{1}(x)-\tilde{M}_{1,1}(x)}{\tilde{M}_{1,1}(x) M_{1}(x)}\right| \rightarrow 0
$$

almost surely as $n \rightarrow \infty$. Then

$$
\begin{aligned}
\sup _{0 \leq x \leq b}\left|\int_{0}^{x} \frac{1}{\tilde{M}_{1,1}(t)} d t-\int_{0}^{x} \frac{1}{M_{1}(t) d t}\right| & \leq \sup _{0 \leq x \leq b}\left\{\int_{0}^{x}\left|\frac{1}{\tilde{M}_{1,1}(x)}-\frac{1}{M_{1}(x)}\right| d x\right\} \\
& \rightarrow 0 \text { almost surely as } n \rightarrow \infty
\end{aligned}
$$

Then we have

$$
\begin{aligned}
& \sup _{0 \leq x \leq b}\left|\tilde{S}_{1,1}(x)-S_{1}(x)\right| \\
= & \sup _{0 \leq x \leq b}\left|\frac{\tilde{M}_{1,1}(0)}{\tilde{M}_{1,1}(x)} \exp \left\{-\int_{0}^{x} \frac{1}{\tilde{M}_{1,1}(x)} d t\right\}-\frac{M(0)}{M(x)} \exp \left\{-\int_{0}^{x} \frac{1}{M(x)} d t\right\}\right| \\
\rightarrow & 0 \text { almost surely as } n \rightarrow \infty .
\end{aligned}
$$

Hence we have proved the consistency of $\tilde{S}_{1,1}(x)$ and $\tilde{M}_{1,1}(x)$ over $[0, b]$.

\subsection{Consistency for $\tilde{M}_{1,2}$ and $\tilde{S}_{1,2}$}

Now we will show the consistency for $\tilde{S}_{1,2}(x)$ and $\tilde{M}_{1,2}(x)$ under the condition that $X \leq_{m r l} Y$. First, we establish the following theorem.

Theorem 4.2 Let $S_{1}(x)$ and $S_{2}(x)$ be continuous with finite means. Then as $\lambda_{n} \rightarrow \infty$, for any $b<\infty$,

$$
\sup _{0 \leq x \leq b}\left|\tilde{\theta}_{n}(x)-\theta(x)\right| \rightarrow 0 \text { almost surely as } n \rightarrow \infty \text {. }
$$

Proof: To prove the above theorem, we need the following lemma, introduced in Rojo and Samaniego(1993) as Lemma 1. 
Lemma 4.1 let $h$ and $g$ be bounded functions on interval $[0, x]$, then

$$
\left|\inf _{0 \leq y \leq x} h(y)-\inf _{0 \leq y \leq x} g(y)\right| \leq \sup _{0 \leq y \leq x}|h(y)-g(y)|
$$

Now, for any $x \in[0, b], \quad b<B=\min \left(b_{1}, b_{2}\right)$,

$$
\begin{aligned}
& \sup _{0 \leq x \leq b}\left|\hat{\theta}_{n}(x) \int_{x}^{\infty} S_{2}(u) d u-\theta(x) \int_{x}^{\infty} S_{2}(u) d u\right| \\
= & \sup _{0 \leq x \leq b}\left|\inf _{0 \leq t \leq x} \frac{\int_{t}^{\infty} \hat{S}_{1}(u) d u}{\int_{t}^{\infty} S_{2}(u) d u} \int_{x}^{\infty} S_{2}(u) d u-\inf _{0 \leq t \leq x} \frac{\int_{t}^{\infty} S_{1}(u) d u}{\int_{t}^{\infty} S_{2}(u) d u} \int_{x}^{\infty} S_{2}(u) d u\right| \\
\leq & \sup _{0 \leq x \leq b} \sup _{0 \leq t \leq x}\left|\frac{\int_{t}^{\infty} \hat{S}_{1}(u) d u}{\int_{t}^{\infty} S_{2}(u) d u} \int_{x}^{\infty} S_{2}(u) d u-\frac{\int_{t}^{\infty} S_{1}(u) d u}{\int_{t}^{\infty} S_{2}(u) d u} \int_{x}^{\infty} S_{2}(u) d u\right| \\
= & \sup _{0 \leq x \leq b} \sup _{0 \leq t \leq x}\left|\frac{\int_{x}^{\infty} S_{2}(u) d u}{\int_{t}^{\infty} S_{2}(u) d u}\right|\left|\int_{t}^{\infty} \hat{S}_{1}(u) d u-\int_{t}^{\infty} S_{1}(u) d u\right| \\
\leq & \sup _{0 \leq t \leq x}\left|\int_{t}^{\infty} \hat{S}_{1}(u) d u-\int_{t}^{\infty} S_{1}(u) d u\right| \\
\rightarrow & 0 \operatorname{las}_{n} n \rightarrow .
\end{aligned}
$$

The last step follows from a Lemma A in Barlow et al. (1972), (p. 237) which implies that

$$
\int_{x}^{\infty} S_{1 n}(t) d t \rightarrow \int_{x}^{\infty} S_{1}(t) d t, \forall t>0
$$

Hence, we get

$$
\sup _{0 \leq x \leq b}\left|\hat{\theta}_{n}(x)-\theta(x)\right| \rightarrow 0 \text { as } n \rightarrow \infty
$$

By the definition, $\hat{\theta}_{n}(x)$ is bounded, hence by using the Lemma 3.1(Hille's Lemma), for $\tilde{\theta}_{n}(x)$, we can complete the proof along the similar lines as those of Theorem 4.4.

For the estimator under the reverse ordering we can establish the similar result as above by the Lemma 3.1 and Lemma 2 in Rojo and Samaniego (1993).

Now we present the analysis of $\frac{d}{d x} \tilde{\theta}_{n}(x)$, defined in Eq. (??). 
Theorem 4.3 Let $S_{1}(x)$ and $S_{2}(x)$ be continuous with finite means. Let $\theta(x)$ be twice finitely differentiable, then as $\lambda_{n} \rightarrow \infty$, for any $b<\infty$, we will have

$$
\sup _{0 \leq x \leq b}\left|\frac{d}{d x} \tilde{\theta}_{n}(x)-\frac{d}{d x} \theta(x)\right| \rightarrow 0 \text { almost surely as } n \rightarrow \infty .
$$

Proof: From Eq. (3.13) we could write $\frac{d}{d x} \tilde{\theta}_{n}(x)$ as

$$
\begin{aligned}
\frac{d}{d x} \tilde{\theta}_{n}(x)= & -\lambda_{n}\left\{\sum_{k=0}^{\infty}\left[\theta\left(\frac{k}{\lambda_{n}}\right)-\theta\left(\frac{k+1}{\lambda_{n}}\right)\right] e^{-\lambda_{n} x} \frac{\left(\lambda_{n} x\right)^{k}}{k !}\right. \\
& \left.+\sum_{k=0}^{\infty}\left[\left(\hat{\theta}_{n}\left(\frac{k}{\lambda_{n}}\right)-\hat{\theta}_{n}\left(\frac{k+1}{\lambda_{n}}\right)\right)-\left(\theta\left(\frac{k}{\lambda_{n}}\right)-\theta\left(\frac{k+1}{\lambda_{n}}\right)\right)\right] e^{-\lambda_{n} x} \frac{\left(\lambda_{n} x\right)^{k}}{k !}\right\} \\
= & T_{n 1}(x)+T_{n 2}(x) .
\end{aligned}
$$

For establishing the convergence of $T_{n 1}(x)$, we expand $\theta(x)$ as a Taylor Series at $\frac{k}{\lambda_{n}}$.

$$
\theta(x)=\theta\left(\frac{k}{\lambda_{n}}\right)+\theta^{\prime}\left(\frac{k}{\lambda_{n}}\right)\left(x-\frac{k}{\lambda_{n}}\right)+\frac{1}{2} \theta^{\prime \prime}\left(\frac{k}{\lambda_{n}}\right)\left(x-\frac{k}{\lambda_{n}}\right)^{2}+o\left(x-\frac{k}{\lambda_{n}}\right)^{-2}
$$

If we replace $x$ by $\frac{k+1}{\lambda_{n}}$, we could write

$$
-\lambda_{n}\left[\theta\left(\frac{k}{\lambda_{n}}\right)-\theta\left(\frac{k+1}{\lambda_{n}}\right)\right]=\theta^{\prime}\left(\frac{k}{\lambda_{n}}\right)+\frac{1}{2 \lambda_{n}} \theta^{\prime \prime}\left(\frac{k}{\lambda_{n}}\right)+o\left(\frac{1}{\lambda_{n}}\right)
$$

so that under the assumed boundedness of the first two derivatives of $\theta(x)$, we may virtually repeat the proof of Theorem 3.1 and conclude that

$$
\sup _{0 \leq x \leq b}\left|T_{n 1}(x)-\frac{d}{d x} \theta(x)\right| \rightarrow 0, \text { almost surely as } \lambda_{n} \rightarrow \infty .
$$

Next, we will show $\sup _{0 \leq x \leq b}\left|T_{n 2}(x)\right| \rightarrow 0$ as $n \rightarrow \infty$. Note that for large $\lambda_{n}$, by Hille's theorem

$$
T_{n 2}(x) \approx-\lambda_{n}\left\{\left[\hat{\theta}_{n}\left(x+\frac{1}{\lambda_{n}}\right)-\hat{\theta}_{n}(x)\right]-\left[\theta_{n}\left(x+\frac{1}{\lambda_{n}}\right)-\theta(x)\right]\right\} .
$$

Since the right hand of the above expression converges almost surely to the

$$
\lim _{h_{n} \rightarrow 0}\left[\left(\frac{\theta(x+h)-\theta(x)}{h}\right)-\left(\frac{\theta(x+h)-\theta(x)}{h}\right)\right] .
$$


This completes the proof.

Writing $S_{1}(x)$ as

$$
S_{1}(x)=\theta(x) S_{2}(x)-\frac{d}{d x} \theta(x) \int_{x}^{\infty} S_{2}(u)
$$

we can show that

$$
\begin{aligned}
& \sup _{0 \leq x \leq b}\left|\tilde{S}_{12}(x)-S_{1}(x)\right| \\
= & \sup _{0 \leq x \leq b}\left|-\left[\frac{d}{d x} \tilde{\theta}_{n}(x)-\frac{d}{d x} \theta(x)\right] \int_{x}^{\infty} S_{2}(u) d u+S_{2}(x)\left[\tilde{\theta}_{n}(x)-\theta(x)\right]\right| \\
\leq & \sup _{0 \leq x \leq b}\left|\frac{d}{d x} \tilde{\theta}_{n}(x)-\frac{d}{d x} \theta(x)\right| \int_{x}^{\infty} S_{2}(u) d u+\sup _{0 \leq x \leq b}\left|\tilde{\theta}_{n}(x)-\theta(x)\right| S_{2}(x) \\
\rightarrow & 0 \text { almost surely as } n \rightarrow \infty,
\end{aligned}
$$

by using Theorems 4.2 and 4.3 .

Furthermore, by continuous mapping theorem, we see that

$$
\sup _{0 \leq x \leq b}\left|\tilde{M}_{1,2}(x)-M_{1}(x)\right| \rightarrow 0 \text { almost surely as } n \rightarrow 0
$$

for every $b$ less than the support of $\tilde{S}_{1,2}(x)$, where $\tilde{M}_{1,2}(x)$ is defined as (??). Hence, we obtain the following theorem proving strong consistency of $\tilde{S}_{1,2}$ and that of $\tilde{M}_{1,2}$.

Theorem 4.4 Let $S_{1}(x), S_{2}(x)$ be continuous a.e with support on $[0, \infty), \lambda_{n} \rightarrow \infty$, a.s. then,

(i) for any $b<\infty$,

$$
\sup _{0 \leq x \leq b}\left|\tilde{M}_{1,2}(x)-M_{1}(x)\right| \rightarrow 0 \text { almost surely as } n \rightarrow \infty,
$$

(ii) and

$$
\sup _{0 \leq x \leq b}\left|\tilde{S}_{1,2}(x)-S_{1}(x)\right| \rightarrow 0 \text { almost surely as } n \rightarrow \infty \text {. }
$$

Proof of the strong convergence of the estimators in the two sample case parallels to that in the one sample case and is therefore omitted. In the next section we present a simulation study comparing the two estimators. 
Table 1: Comparison of Bias and MSE of $M_{1}^{\star}, \tilde{M}_{1,1}$ and $\tilde{M}_{1,2}$ at various $q$-quantiles

\begin{tabular}{|c|c|c|c|c|c|c|c|c|c|c|}
\hline & \multicolumn{5}{|c|}{$n=10$} & \multicolumn{5}{|c|}{$n=20$} \\
\hline & \multicolumn{3}{|c|}{ Bias } & \multirow[b]{2}{*}{$R E_{1,1}$} & \multirow[b]{2}{*}{$R E_{1,2}$} & \multicolumn{3}{|c|}{ Bias } & \multirow[b]{2}{*}{$R E_{1,1}$} & \multirow[b]{2}{*}{$R E_{1,2}$} \\
\hline$q$ & $M_{1}^{*}$ & $\tilde{M}_{1,1}$ & $\tilde{M}_{1,2}$ & & & $M_{1}^{*}$ & $\tilde{M}_{1,1}$ & $\tilde{M}_{1,2}$ & & \\
\hline \multicolumn{11}{|c|}{$M_{1}(x)=\frac{1}{3}(1-x), M_{2}(x)=\frac{1}{2}(1-x)$} \\
\hline 0.1 & -0.0395 & -0.0368 & -0.03980 & 1.2178 & 1.0787 & -0.0271 & -0.0260 & -0.0260 & 1.0997 & 1.0053 \\
\hline 0.2 & -0.0367 & -0.0340 & -0.0355 & 1.3019 & 1.1395 & -0.0279 & -0.0261 & -0.0252 & 1.1845 & 0.9160 \\
\hline 0.5 & -0.0266 & -0.0272 & -0.0477 & 1.2578 & 0.7249 & -0.0206 & -0.0209 & -0.0258 & 1.2919 & 1.0157 \\
\hline 0.8 & -0.0311 & -0.0025 & -0.0211 & 4.0131 & 4.8582 & -0.0112 & -0.0036 & -0.0032 & 2.8544 & 1.0551 \\
\hline 0.9 & -0.0268 & -0.0181 & -0.0070 & 1.7160 & 23.3061 & -0.0137 & 0.0122 & -0.0077 & 2.1733 & 8.1841 \\
\hline \multicolumn{11}{|c|}{$M_{1}(x)=1, M_{2}(x)=1.1$} \\
\hline 0.1 & -0.0958 & -0.1142 & -0.1005 & 0.9265 & 0.9779 & -0.0637 & -0.0718 & -0.0680 & 0.9552 & 0.9476 \\
\hline 0.2 & -0.1028 & -0.1253 & -0.1117 & 1.0145 & 1.0556 & -0.0703 & -0.0806 & -0.0761 & 1.0120 & 0.9930 \\
\hline 0.5 & -0.1440 & -0.1686 & -0.1601 & 1.1112 & 1.1107 & -0.1211 & -0.1303 & -0.1254 & 1.0824 & 1.0635 \\
\hline 0.8 & -0.3076 & -0.3095 & -0.2805 & 1.5642 & 1.9126 & -0.1917 & -0.2207 & -0.2101 & 1.1993 & 1.2830 \\
\hline 0.9 & -0.5337 & -0.4811 & -0.3905 & 1.5824 & 2.4140 & -0.3738 & -0.3414 & -0.3031 & 1.6022 & 2.0353 \\
\hline \multicolumn{11}{|c|}{$M_{1}(x)=\frac{1}{2} x+1, M_{2}(x)=x+1$} \\
\hline 0.1 & -0.2131 & -0.2278 & -0.2262 & 0.9743 & 0.9959 & -0.1265 & -0.1377 & -0.1390 & 0.9482 & 0.9004 \\
\hline 0.2 & -0.2097 & -0.2423 & -0.2308 & 0.9167 & 0.9506 & -0.1427 & -0.1456 & -0.1340 & 0.9882 & 0.9338 \\
\hline 0.5 & -0.2602 & -0.2949 & -0.2493 & 0.9238 & 0.8701 & -0.1701 & -0.1912 & -0.1530 & 0.9197 & 0.8342 \\
\hline 0.8 & -0.5984 & -0.6266 & -0.4877 & 1.1967 & 1.1432 & -0.3333 & -0.4471 & -0.3727 & 1.0685 & 1.0292 \\
\hline 0.9 & -1.1794 & -1.1569 & -0.9377 & 1.2524 & 1.4528 & -0.7107 & -0.7718 & -0.6625 & 1.2238 & 1.2153 \\
\hline
\end{tabular}

Note: $R E_{1,1}=\operatorname{MSE}\left(M_{1}^{\star}\right) / \operatorname{MSE}\left(\tilde{M}_{1,1}\right), R E_{1,2}=\operatorname{MSE}\left(M_{1}^{\star}\right) / \operatorname{MSE}\left(\tilde{M}_{1,2}\right.$. 
Table 2: Comparison of bias and MSE of $\tilde{S}_{1,1}(x)$ and $\tilde{S}_{1,2}(x)$ at various $q$-quantiles

\begin{tabular}{|c|c|c|c|c|c|c|c|c|}
\hline & \multicolumn{4}{|c|}{$n=10$} & \multicolumn{4}{|c|}{$n=20$} \\
\hline & \multicolumn{2}{|c|}{ Bias } & \multicolumn{2}{|c|}{ MSE } & \multicolumn{2}{|c|}{ Bias } & \multicolumn{2}{|c|}{ MSE } \\
\hline$q$ & $\tilde{S}_{1,1}$ & $\tilde{S}_{1,2}$ & $\tilde{S}_{1,1}$ & $\tilde{S}_{1,2}$ & $\tilde{S}_{1,1}$ & $\tilde{S}_{1,2}$ & $\tilde{S}_{1,1}$ & $\tilde{S}_{1,2}$ \\
\hline \multicolumn{9}{|c|}{$M_{1}(x)=\frac{1}{2}(1-x), M_{2}(x)=M_{2}(x)=(1-x)$} \\
\hline 0.1 & -0.0126 & -0.0091 & 0.0026 & 0.0037 & 0.0003 & 0.0008 & 0.0008 & 0.0011 \\
\hline 0.2 & -0.0264 & -0.0235 & 0.0058 & 0.0062 & -0.0068 & -0.0078 & 0.0017 & 0.0019 \\
\hline 0.5 & -0.0507 & -0.0285 & 0.0096 & 0.0093 & -0.0336 & -0.0235 & 0.0044 & 0.0044 \\
\hline 0.8 & -0.0652 & -0.0820 & 0.0067 & 0.0085 & -0.0474 & -0.0519 & 0.0036 & 0.0042 \\
\hline 0.9 & -0.0403 & -0.0582 & 0.0025 & 0.0037 & -0.0345 & -0.0477 & 0.0017 & 0.0026 \\
\hline \multicolumn{9}{|c|}{$M_{1}(x)=1, M_{2}(x)=1.1$} \\
\hline 0.1 & 0.0114 & -0.0040 & 0.0047 & 0.0057 & 0.0103 & 0.0063 & 0.0019 & 0.0019 \\
\hline 0.2 & 0.0234 & 0.0090 & 0.0081 & 0.0068 & 0.0231 & 0.0192 & 0.0038 & 0.0038 \\
\hline 0.5 & 0.0347 & 0.0296 & 0.0102 & 0.0097 & 0.0043 & 0.0029 & 0.0042 & 0.0043 \\
\hline 0.8 & 0.0481 & 0.0414 & 0.0069 & 0.0067 & 0.0193 & 0.0182 & 0.0029 & 0.0030 \\
\hline 0.9 & 0.0383 & 0.0310 & 0.0050 & 0.0045 & 0.0202 & 0.0183 & 0.0018 & 0.0018 \\
\hline \multicolumn{9}{|c|}{$M_{1}(x)=\frac{1}{2} x+1, M_{2}(x)=x+1$} \\
\hline 0.1 & 0.0094 & 0.0052 & 0.0043 & 0.0075 & -0.0003 & 0.0009 & 0.0038 & 0.0050 \\
\hline 0.2 & 0.0094 & -0.0043 & 0.0075 & 0.0081 & -0.0111 & -0.0187 & 0.0060 & 0.0074 \\
\hline 0.5 & 0.0074 & -0.0081 & 0.0106 & 0.0101 & -0.0059 & -0.0153 & 0.0063 & 0.0073 \\
\hline 0.8 & 0.0388 & 0.0314 & 0.0064 & 0.0058 & 0.0117 & 0.0100 & 0.0022 & 0.0022 \\
\hline 0.9 & 0.0377 & 0.0341 & 0.0039 & 0.0035 & 0.0180 & 0.0184 & 0.0012 & 0.0012 \\
\hline
\end{tabular}




\section{A Simulation Study to Compare the Two Estimators}

This section presents some simulation results comparing the estimator of $\mathrm{Hu}$ et al. (2002) and the estimators proposed here for various quantiles. Since, Hu et al. (2002) do not present any results for estimating the survival function we compare the two estimators of survival functions also. The following decreasing, constant and increasing MRL functions are used to carry out the simulation:

$$
M_{i}(x)=a_{i}\left(1-\frac{x}{b_{i}}\right) I\left[x \leq b_{i}\right], \quad b_{i}>a_{i}, \text { with } S_{i}(x)=\left(1-\frac{x}{b_{i}}\right)^{\frac{b_{i}}{a_{i}}-1}
$$

which corresponds to the $U(0,1)$ distribution when $a_{i}=0.5, b_{i}=1$;

$$
\begin{gathered}
M_{i}(x)=\theta_{i} \text { corresponding to the } \exp \left(\theta_{i}\right) \text { distribution ; and } \\
M_{i}(x)=a_{i} x+b_{i}, \quad a_{i} x+b_{i} \geq 0, \quad b_{i}>0 \text { with } S_{i}(x)=\left(\frac{a_{i} x+b}{b_{i}}\right)^{-\left(1+\frac{1}{a_{i}}\right)} .
\end{gathered}
$$

Our interest is in contrasting the simulated bias and MSE. The results are for comparison purpose only and we have used only 1000 replication for samples of size 10 and 20.

Table 1 compares the bias and MSE of the smooth estimators proposed here for the MRLF where as Table 2 explores the same for the corresponding smooth estimators of the survival functions. Three sets of MRLF's are considered, i $M_{1}(x)=\frac{1}{3}(1-x), M_{2}(x)=\frac{1}{2}(1-x)$, with corresponding survival functions $S_{1}(x)=(1-x)^{2}$ and $S_{2}(x)=(1-x)$ depicting decreasing MRL, (ii) $M_{1}(x)=1, M_{2}(x)=1.1$, with corresponding survival functions $S_{1}(x)=e^{-x}$ and $S_{2}(x)=e^{-\frac{x}{1.1}}$ depicting constant MRL and (iii) $M_{1}(x)=\frac{1}{2} x+1, M_{2}(x)=x+1$, with responding survival functions $S_{1}(x)=\left(\frac{2}{x+2}\right)^{3}$ and $S_{2}(x)=(x+1)^{-2}$, depicting the increasing MRL.

From these tables, it may be seen, generally that the smooth estimators have a little bit more bias than the un-smooth estimator of $\mathrm{Hu}$ et al. (2002), but almost always they have smaller MSE, particularly in the tail of the distribution. We note from Table 1 that for the decreasing MRL function model, $\tilde{M}_{1,1}(x)$ and $\tilde{M}_{1,2}(x)$ even have smaller estimated bias than $M_{1}^{\star}(x)$ as well as smaller MSE. For the increasing MRL case, our estimators do not seem to work as well as under the decreasing model, their MSE increases as $q$ increases from 0 to 0.5 , but after that, it decreases. That is, in the tails our estimators do perform better.

We would also like to contrast $\tilde{M}_{1,1}, \tilde{S}_{1,1}(x)$ and $\tilde{M}_{1,2}(x), \tilde{S}_{1,2}(\mathrm{x})$. Unfortunately, we can not find any general rules for comparing the two estimators proposed here. It seems though, that the first method generally gives larger bias, especially in the last two models. However, the difference is very very small. And in general, the $\tilde{M}_{1,2}$ produces much smaller MSE in 
the tails of the survival function. But the $\widetilde{S}_{1,2}$ is not always equal to 1 at 0 ; it approaches 1 as $n$ becomes large. We also see the pattern that estimated bias decreases with the increase in sample size, which is expected due to strong consistency result.

Overall, smoothing proposed here does not produce much higher bias in the estimators of MRLFs and survival functions and may produce smaller MSE's in some cases. Out of the two smoothing methods proposed here for preserving the MRL ordering, Method 1 is simpler to use. It may give larger bias than the second method, but the difference may not be significant, hence Method 1 may be preferred in practical applications.

\section{References}

[1] Abdous, B. and Berred, A. (2005). Mean residual life estimation. Journal of Statistical Planning and Inference 132 3-19.

[2] Barlow, R.E., Bartholomew, D.J., Bremner, J.M. and Brunk, H.D. (1972). Statistical Inference under Order Restrictions. Wiley, new York.

[3] Chaubey, Y.P. and Kochar, S.C. (2000). Smooth estimation of stochastically ordered survival functions. Journal of Indian Statistical Association 38, 209-225.

[4] Chaubey, Y. P. and Sen, P. K. (1996). On smooth estimation of survival and density functions. Statistics and Decisions 14, 1-22.

[5] Chaubey, Y. P. and Sen, P. K. (1998). On smooth functional estimation under random censorship. In Frontiers in Reliability, vol. 4, Series on Quality, Reliability and Engineering Statistics, Ed.: A.P. Basu et al., Singapore: World Scientific Publishing Co. Pvt. Ltd., 83-97.

[6] Chaubey, Y. P. and Sen, P. K. (1999). On smooth estimation of mean residual life. Journal of Statistical Planning and Inference 75 223-236.

[7] Csörgo, M. and Zitikis, R. (1996). Mean residual life process. Annals of Statistics 24 1717-1739.

[8] Ebrahimi, N. (1993). Estimation of two ordered mean residual life functions. Biometrics 49, 409-417.

[9] Embrechts, P., Kluppelberg, C. and Mikosch, T. (1997). Modelling Extremal Events. Ed. I. Karatzas and M. Yor, Springer, Berlin. 
[10] Feller, W. (1965). An Introduction to Probability Theory and its Applications, Vol. II. John Wiley and Sons, New York.

[11] Guess, F. and Proschan, F. (1988). Mean residual life, theory and applications. In Handbook of Statistics, Vol. 7, Reliability and Qualiy Control.Eds.: P.R. Krishnaiah and C.R. Rao, 215-224.

[12] Hille, E. (1948). Functional Analysis and Semigroups. Am. Math. Soc. Colloq. Pub., 31, American Mathematical Society, New York.

[13] Hu, X. Kochar, S.C., Mukerjee, H. and Samniego, F. (2002). Estimation of two ordered mean residual life functions. Journal of Statistical Planning and Inference 107 321-341.

[14] Kim, J. S. and Proschan, F. (1991). Piecewise exponential estimator of the survivor function. IEEE Trans. Reliability 40, 134-139.

[15] Mukerjee, H. (1996). Estimation of survival functions under uniform stochastic ordering. J. Amer. Statist. Assoc. 91, 1684-1689.

[16] Ruiz, J.M. and Guillammon, A. (1996). Nonparametric recursive estimator of mean residual life and vitality parameter under mixing dependent conditions. Communications in Statistics-Theory and Methods 4 1999-2011.

[17] Rojo, J. and Samaniego, F. J. (1993). On estimating a survival curve subject to a uniform stochastic ordering constraint. J. Amer. Statist. Assoc. 88, 566-572.

[18] Shaked, M. and Shanthikumar, J. (1994). Stochastic Orders and Their Applications. Academic Press, New York.

[19] Yang, G. L. (1978).Estimation of a biometric function. Ann. Statist. 6 112-116. 


\section{List of Recent Technical Reports}

46. Shuanming Li and José Garrido, On the Time Value of Ruin for a Sparre Anderson Risk Process Perturbed by Diffusion, November 2003

47. Yogendra P. Chaubey, Cynthia M. DeSouza and Fassil Nebebe, Bayesian Inference for Small Area Estimation under the Inverse Gaussian Model via Cibbs Sampling, December 2003

48. Alexander Melnikov and Victoria Skornyakova, Pricing of EquityLinked Life Insurance Contracts with Flexible Guarantees, May 2004

49. Yi Lu and José Garrido, Regime-Switching Periodic Models for Claim Counts, June 2004.

50. I. Urrutia-Romaní, R. Rodríguez-Ramos, J. Bravo-Castillero and R. Guinovart-Díaz, Asymptotic Homogenization Method Applied to Linear Viscoelastic Composites. Examples, August 2004.

51. Yi Lu and José Garrido, Double Periodic Non-Homogeneous Poisson Models for Hurricanes Data, September 2004.

52. M.I. Beg and M. Ahsanullah, On Characterizing Distributions by Conditional Expectations of Functions of Generalized Order Statistics, September, 2004.

53. M.I. Beg and M. Ahsanullah, Concomitants of Generalized Order Statistics from Farlie-Gumbel-Morgenstern Distributions, September, 2004.

54. Yogendra P. Chaubey and Debaraj Sen, An investigation into properties of an estimator of mean of an inverse Gaussian population, September, 2004.

55. Steven N. Evans and Xiaowen Zhou, Balls-in-boxes duality for coalescing random walks and coalescing Brownian motions, September, 2004.

56. Qihe Tang, Asymptotic ruin probabilities of the renewal model with constant interest force and regular variation, November, 2004.

57. Xiaowen Zhou, On a classical risk model with a constant dividend barrier, November, 2004. 
58. K. Balasubramanian and M.I. Beg, Three isomorphic vector spaces-II, December, 2004.

59. Michael A. Kouritzin and Wei Sun, Rates for branching particle approximations of continuous-discrete filters, December, 2004.

60. Rob Kaas and Qihe Tang, Introducing a dependence structure to the occurrences in studying precise large deviations for the total claim amount , December, 2004.

61. Qihe Tang and Gurami Tsitsiashvili, Finite and infinite time ruin probabilities in the presence of stochastic returns on investments, December, 2004.

62. Alexander Melnikov and Victoria Skornyakova, Efficient hedging methodology applied to equity-linked life insurance, February, 2005.

63. Qihe Tang, The finite time ruin probability of the compound Poisson model with constant interest force, June, 2005.

64. Marc J. Goovaerts, Rob Kaas, Roger J.A. Laeven, Qihe Tang and Raluca Vernic, The tail probability of discounted sums of Pareto-like losses in insurance, August, 2005.

65. Yogendra P. Chaubey and Haipeng Xu, Smooth estimation of survival functions under mean residual life ordering, August, 2005.

Copies of technical reports can be requested from:

Prof. Xiaowen Zhou

Department of Mathematics and Statistics

Concordia University

7141, Sherbrooke Street West

Montréal (QC) H4B 1R6 CANADA 